\title{
HEARTLIGHT - ACQUISITION TIMES FOR A NOVEL FOREHEAD HEART RATE SENSOR IN DELIVERY ROOM RESUSCITATION OF PRETERM INFANTS
}

\author{
C. Mann ${ }^{1}$, C. Ward ${ }^{1}$, J. Teoh ${ }^{2}$, M. Grubb ${ }^{2}$, J. Crowe $^{2}$, B. Hayes-Gill ${ }^{2}$, N. Marlow ${ }^{3}$, D. Sharkey ${ }^{1}$
}

${ }^{I}$ Division of Academic Child Health, ${ }^{2}$ Electrical and Electronic Engineering, University of Nottingham, Nottingham, ${ }^{3}$ Institute for Women's Health, University College London, London, UK

Background: Approximately $10 \%$ of newborn infants require some resuscitation at birth and heart rate (HR) is considered the best indicator of effective resuscitation. Current HR assessment, using a stethoscope, is non-continuous, can interrupt resuscitation and is estimated incorrectly in $20-30 \%$ of cases. Pulse oximetry (PO) is not specifically designed for newborn HR monitoring and can be unreliable in low perfusion states. A simple forehead HR sensor would be advantageous allowing quick placement, continuous monitoring and improved reliability in low perfusion states.

Aims: Further development of a user friendly, quick and reliable forehead HR sensor for use during resuscitation of preterm infants in the delivery room.

Methods: Our novel forehead HR sensor (HeartLight), utilises patented reflectance photoplethysmography (PPG), to rapidly detect HR and can be sited in $\sim 5$ seconds. We investigated acquisition time of HeartLight versus PO at birth in preterm infants in the delivery room (gestation $32+6$ weeks \pm 20 days). Time to acquire a reliable signal was measured from the time each sensor was applied.

Results: Median time to obtain 1 and 2 seconds of PPG signal were calculated (table).

\begin{tabular}{|c|c|c|}
\hline Device (n=10) & Time for 1 second (median+IQR) & $\begin{array}{c}\text { Time for 2 seconds } \\
\text { (median+IQR) }\end{array}$ \\
\hline \hline HeartLight & $5.8(3.1-9.7) \mathrm{s}$ & $8.1(6.4-18.8) \mathrm{s}$ \\
\hline Masimo PO & $10.6(8.1-17.0) \mathrm{s}$ & $18.2(9.6-29.9) \mathrm{s}$ \\
\hline
\end{tabular}

[Acquisition times]

Conclusion: HeartLight rapidly detects a pulsatile signal in newborn preterm infants, more quickly than PO. HeartLight may offer a rapid, real time monitoring solution for HR during delivery room resuscitation and is undergoing further clinical trials.

Funding: MRC DPFS portfolio award 